\title{
Análisis de la actividad investigadora en arquitectura y urbanismo según los resultados de la CNEAI
}

\author{
Arianna Guardiola-Víllora*, Luisa Basset-Salom*
}

Resumen: Los sexenios de investigación, creados inicialmente como complemento retributivo, se han convertido en un indicador de calidad imprescindible para el personal docente e investigador de las universidades españolas. Con objeto de conocer la situación actual de la investigación reconocida por el Ministerio de Educación en las áreas relacionadas con el estudio de la Arquitectura y el Urbanismo, en este trabajo se analizan los resultados de la última evaluación de la CNEAI, en aquellas áreas de conocimiento del campo 6.3 "Arquitectura, Ingeniería Civil, Construcción y Urbanismo", que se han considerado áreas de investigación de los arquitectos profesores numerarios de universidad. De este trabajo se deduce la necesidad de desarrollar nuevas estrategias que contribuyan a dar mayor visibilidad y reconocimiento a la labor investigadora realizada por los arquitectos, que dista mucho de la oficialmente reconocida.

Palabras clave: CNEAI, investigación universitaria, sexenios, producción científica, arquitectura y urbanismo.

\section{Research activity in architecture and urbanism: an analysis based on CNEAl results}

Abstract: Research sexennials, which originated for salary complements, have become an essential quality indicator for Spanish academics. In order to understand the current state of research recognised by the Ministry of Education in areas related to the study of architecture and urbanism, this article analysed the results of the latest CNEAI evaluation of those areas within field 6.3 considered to be research topics of professors of architecture: Architecture, Civil Engineering, Construction and Urbanism. The conclusion is that new strategies are needed in order to attain greater visibility and recognition than that officially recognized, for the research produced by architects.

Keywords: CNEAI, academic research, sexennials, scholarly production, architecture and urbanism.

* Universitat Politècnica de València: Escuela Técnica Superior de Arquitectura. Dept. Mecánica de Medios Continuos y Teoría de Estructuras. Valencia. Correo-e: aguardio@mes.upv.es; ibasset@mes.upv.es.

Recibido: 14-11-2011; 2. ${ }^{a}$ versión: 24-02-2012; aceptado: 3-03-2012. 


\section{Introducción}

Con objeto de incentivar la actividad investigadora del profesorado universitario se introdujo, en 1989 (Real Decreto 1086/1989, de 28 de agosto) un complemento retributivo que premiaba la producción científica de aquellos profesores que voluntariamente lo solicitaban, siendo una Comisión Nacional, CNEAI, (Comisión Nacional Evaluadora de la Actividad Investigadora) integrada por representantes del Ministerio de Educación y Ciencia y de las Comunidades Autónomas con competencias asumidas en materia universitaria, la encargada de la evaluación en materia de investigación científica y técnica.

A partir de ese momento, la evaluación de la actividad científica será un proceso reglado, estableciéndose, en sucesivas Ordenes del Ministerio de Educación y Ciencia y Resoluciones de la Presidencia de la CNEAI, la constitución de los comités evaluadores, los criterios de calidad a considerar en la evaluación así como quién, cuándo y cómo se puede solicitar. El establecimiento de este sistema de evaluación científica ha generado un incremento de la producción científica española o cuando menos ha propiciado su internacionalización y visibilidad (Sanz-Menéndez 1995; Jiménez-Contreras y otros, 2003), aunque no por ello está exento de polémica, debatiéndose, desde entonces y todavía hoy, la selección, adecuación, aplicación e influencia en las diferentes áreas de conocimiento (De las Heras y Polo, 2009, Delgado-López-Cózar, 2009; Jiménez-Contreras y otros, 2011).

En diciembre de 2001, la publicación de la Ley Orgánica de Universidades (LOU), introduce en el sistema universitario español "mecanismos externos de evaluación de calidad, conforme a criterios objetivos y procedimientos transparentes". En esa misma ley se contempla la creación de la ANECA (Agencia Nacional de Evaluación y Acreditación), para desarrollar de manera independiente dicha actividad evaluadora, adoptando en la práctica los mismos criterios establecidos por la CNEAI. A partir de este momento, el sexenio, incentivo que premiaba la actividad investigadora, empieza a considerarse indicador de calidad.

Cuatro años después, "habiendo incrementado la Comisión Nacional de manera notable su experiencia en la evaluación y habiéndose convertido sus evaluaciones (sexenios) en referencia para diversas tareas relativas a la toma de decisiones en politicas de personal de instituciones científicas" la CNEAI desarrolla unos criterios de objetivación formal indicativos de la calidad de la investigación que han ido consolidándose en el proceso evaluador, publicando, además, en la Resolución de 25 de octubre de 2005, los criterios específicos de evaluación por campos científicos, que seguirán perfilándose en cada convocatoria anual (RuizPérez y otros, 2010).

Finalmente, La Ley Orgánica 4/2007, de 12 de abril, por la que se modifica la LOU establece como requisito imprescindible para el acceso a los cuerpos docentes universitarios la acreditación nacional por parte de la ANECA y reduce los cuerpos de funcionarios docentes universitarios a Catedráticos de Universidad (CU) y Titulares de Universidad (TU), suprimiendo los cuerpos de Catedráticos 
de Escuela Universitaria (CEU) y Titulares de Escuela Universitaria (TEU), contemplándose, en las disposiciones adicionales de dicha ley, la integración de los cuerpos a extinguir al cuerpo de Titulares de Universidad, en el caso de los CEU, automáticamente, previa solicitud, y en el de los TEU, tras la acreditación, en la que se valorará la investigación, la gestión y, particularmente, la docencia.

Es evidente que, el sexenio de investigación es cada vez más valorado, no tanto por el complemento retributivo que supone, sino por haberse convertido en un indicador de calidad y de reconocimiento, siendo un requisito imprescindible para la promoción profesional, para poder optar a proyectos de investigación financiados y acceder a otros recursos derivados de la investigación, para formar parte de ciertas comisiones, pertenecer a Comités y Tribunales académicos, acreditarse a Titular de Universidad o ser nombrado profesor emérito, entre otros.

La investigación en arquitectura y urbanismo tiene un carácter específico y constituye un tema controvertido (Monedero-Gil, 2004), ya que la actividad investigadora de un arquitecto no sigue las mismas pautas que las de un matemático, químico o biólogo, y no es fácil medirla científicamente o en términos de innovación. Según Helio Piñón, Catedrático de Proyectos de Arquitectura de la Universitat Politécnica de Catalunya (Pinón-Pallarés, 2007) «investigar no supone necesariamente innovar, sino profundizar en la constitución y el sentido de determinadas obras consideradas de calidad, de modo que el análisis proporcione criterios para concebir, proyectar y construir otras que, si se atienden a sus condiciones peculiares, conseguirán resultados necesariamente renovados».

A pesar de que se han ido produciendo modificaciones en los criterios de evaluación de la calidad investigadora en las áreas de arquitectura y urbanismo, en un intento de acercarse a las peculiaridades de éstas, actualmente, el porcentaje de sexenios no solicitados y rechazados es elevado. La opinión de que estos criterios no se ajustan a gran parte de la investigación desarrollada por los profesores de universidad arquitectos es todavía un tema latente.

En septiembre de 2004, el Instituto Universitario de Ciencias de la Construcción (IUCC) de la Escuela de Arquitectura de Sevilla organizó las primeras jornadas de Investigación en Arquitectura y Urbanismo (I Jornadas IAU), para tratar las grandes diferencias entre la realidad de la investigación en las escuelas de arquitectura españolas y la política científica universitaria de calidad planteada por la LOU. En esas jornadas se llegó a la conclusión de que era necesario plantear un conjunto de baremos y pautas para que los profesores de las escuelas de arquitectura alcanzasen evaluaciones positivas en su actividad investigadora. En esta línea fue la ponencia que en Sevilla presentó el entonces vicerrector de la UPC (Universidad Politécnica de Cataluña), Francisco Fayos, (Fayos-Valles, 2005) proponiendo diversos cambios de criterio en la evaluación de sexenios, o las reflexiones sobre los indicadores de calidad de Joan Font (Font-Comas, 2006), dos años después.

En 2006, en las segundas jornadas sobre Investigación en Arquitectura y Urbanismo (Conclusiones IAU, 2006) seguía manteniéndose la convicción de que el panorama investigador en las escuelas de arquitectura no iba a experimentar 
cambios sensibles en el futuro inmediato y, tres años después, en las terceras jornadas sobre Investigación en Arquitectura y Urbanismo, III IAU, se siguió cuestionando la capacidad del sistema para valorar la actividad del profesorado por considerar que la evaluación seguía siendo una transposición literal del modelo general de las ciencias.

Para mejorar esa situación, en las mismas fechas los directores y subdirectores de las escuelas de arquitectura acordaron que una comisión permanente de subdirectores estudiara la viabilidad de un proyecto para la elaboración de una serie de indicadores de calidad de la actividad científica y técnica de los profesores de arquitectura, con el objetivo de categorizar las revistas españolas y latinoamericanas relacionadas con las diferentes áreas (proyectos, urbanismo, construcción, composición, etc.) y de determinar los congresos relevantes impulsando, de este modo, la difusión y la visibilidad.

El proceso de evaluación de los profesores de Arquitectura para los sexenios y la acreditación para titularidad y cátedra sigue siendo objeto de debate y análisis tanto por parte de la Conferencia de Directores de las Escuelas de Arquitectura Españolas como por parte del colectivo de profesores de dichas escuelas. En junio 2011, los asistentes a las IV Jornadas internacionales sobre investigación en arquitectura y urbanismo (4 IAU+i, 2011) debatieron, una vez más, la problemática a nivel nacional e internacional, abordando el tema del reconocimiento de las publicaciones de arquitectura y su validación como herramientas de referencia y calidad por los organismos evaluadores de la actividad investigadora, manifestando las desigualdades entre las distintas áreas y la escasa presencia de arquitectos en las comisiones evaluadoras.

El descontento generalizado entre el profesorado de las Escuelas de Arquitectura y la sensación de falta de reconocimiento de su actividad investigadora plantea la necesidad de llevar a cabo un análisis cuantitativo de la situación frente a la investigación de los profesores funcionarios que son arquitectos.

\section{Objetivos}

El objetivo de este trabajo es analizar la situación actual de la investigación reconocida por la CNEAI en las áreas de la Arquitectura y el Urbanismo, a partir de los resultados de investigación de los profesores de universidad numerarios de siete de las doce áreas del subcampo 6.3 "Arquitectura, Ingeniería Civil, Construcción y Urbanismo".

El subcampo 6.3 está formado por las doce áreas siguientes: (100) Composición Arquitectónica, (110) Construcciones Arquitectónicas, (300) Expresión Gráfica Arquitectónica, (305) Expresión Gráfica de la Ingeniería, (505) Ingeniería Cartográfica, Geodésica y Fotogrametría, (510) Ingeniería de la Construcción, (525) Ingeniería del Terreno, (530) Ingeniería e Infraestructura de los Transportes, (605) Mecánica de Medios Continuos y Teoría de Estructuras, (715) Proyectos Arquitectónicos, (720) Proyectos de Ingeniería y (815) Urbanística y Ordenación del Territorio. 
Sin embargo, no todas las áreas del subcampo 6.3 pueden considerarse áreas de investigación de los profesores de universidad que son arquitectos, de modo que, excluyendo aquellas directamente vinculadas con la Ingeniería Civil (en las que los investigadores no son habitualmente arquitectos), se han seleccionado como objeto de análisis las siete áreas siguientes: Composición Arquitectónica, (CP); Construcciones Arquitectónicas, (CT); Expresión Gráfica Arquitectónica, (EG); Ingeniería del Terreno, (IT); Mecánica de Medios Continuos y Teoría de Estructuras, (ST); Proyectos Arquitectónicos, (PR), y Urbanística y Ordenación del Territorio, (UR), de modo que este estudio se basa en los resultados de los 1.393 profesores de universidad funcionarios que pertenecen a dichas áreas, y que representan un 2,9\% del total de profesores (47.734) de las 205 áreas evaluadas por la CNEAI.

\section{Material y métodos}

Para este estudio se ha utilizado los datos publicados por la CNEAI tras la evaluación de 2007, una vez concluido el período de reclamaciones, siendo la fecha de referencia diciembre de 2008, no habiéndose publicado posteriormente ninguna otra información. Estos datos corresponden a la información recogida en la base de datos de la CNEAI (BD-CNEAI) a partir del listado del Registro Central de Personal mantenido por el Consejo de Coordinación Universitaria, en su versión de febrero de 2009 (BD-CCU).

En este fichero, disponible en la página web de la CNEAI, se agrupan los profesores de universidad funcionarios de la BD-CNEAI en unos 1500 registros correspondientes a: Área de conocimiento, género y cuerpo, conteniendo la siguiente información: número de profesores, índice S, índice E, sexenios totales, profesores no presentados y profesores con 0 (sexenios rechazados), 1, 2, 3, 4, 5 , y 6 sexenios concedidos. Se trata de una tabla en formato $x l s$ y accesible en la web de la CNEAI.

Estos datos se han completado con los publicados en el "Informe sobre resultados de las evaluaciones de la CNEAI. La situación en 2009", también disponible en la página web de la CNEAI, en el que se indica el origen de los datos y el significado de los índices de calidad iE e is, que miden el éxito relativo en el proceso de evaluación de los individuos o de las colectividades, como sigue:

Para un colectivo determinado, iS está definido como: iS = NSO/NSP siendo NSO el número total de sexenios obtenidos por el colectivo y NSP el número total de sexenios posibles para dicho colectivo. El número de sexenios obtenidos es el que aparece, para cada individuo, en la BD-CNEAI, y se define el número de sexenios posibles, NSP, como la parte entera de (EDAD-27)/6, tomando NSP = 6 si NSP > 6. El número 27 refleja la edad promedio de inicio de la publicación de los resultados de la investigación (EI). Dado que esa edad puede variar entre los diversos campos científicos, en el informe mencionado anteriormente se calcula como depende iS de EI, siendo el comportamiento en la región de interés aproximadamente lineal. 
En cuanto al índice iE, es un indicador binario para cada individuo, de valor $\mathrm{iE}=1$ si iS $\geq 1$, e $\mathrm{iE}=0$ si iS $<1$. Los valores de iE denotan la fracción de un colectivo que ha tenido un éxito completo en las evaluaciones.

Por último, matizar, que a pesar de que en los informes y memorias de la CNEAI, se excluye a los TEU por ser un «cuerpo a extinguir, cuya consideración distorsiona los resultados", se ha decidido incluirlos en este análisis, ya que representan el $40 \%$ del profesorado objeto de estudio, tal y como se puede observar en la figura 1 (frente al 18\% de TEU de Todos los Campos), siendo los principales interesados en conseguir un tramo de investigación que les permita acreditarse a TU.

FIGURA 1

Distribución de profesores por cuerpos

(áreas objeto de estudio y todos los campos)

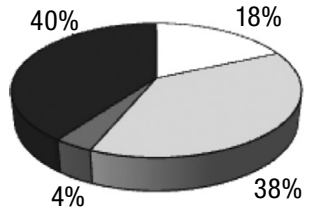

ÁREAS OBJETO DE ESTUDIO

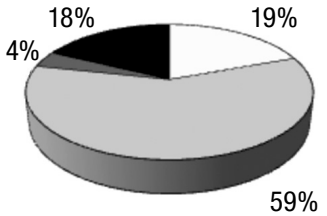

TODOS LOS CAMPOS $\square$ Catedráticos Universidad (CU)

$\square$ Titulares de Universidad (TU)

$\square$ Catedráticos de Escuela Universitaria (CEU)

- Titulares de Escuela Universitaria (TEU)

\section{Resultados}

\subsection{Evaluación de los resultados de la CNEAI en todos los campos y en el campo 6}

En la figura 2 se representa, a partir de la información mencionada (CNEAI, 2005 y 2009), el porcentaje de éxito de los 11 campos científicos durante el período 1989-2007. Estos campos son: Matemáticas y Física (1), Química (2), Biología Celular y Molecular (3), Ciencias Biomédicas (4), Ciencias de la Naturaleza (5), Ingenierías y Arquitectura (6), Ciencias Sociales, Políticas, del Comportamiento y de la Educación (7), Ciencias Económicas y Empresariales (8), Derecho y Jurisprudencia (9) Historia y Expresión Artística (10), Filosofía, Filología y Lingüística (11). En ella se aprecia que, mientras los campos 1, 2 y 3 alcanzan valores superiores al 85\%, el porcentaje de sexenios concedidos sobre los evaluados en el campo 6, que es el que nos ocupa, es inferior a la media.

Haciendo un estudio de la evolución del éxito obtenido por los profesores en el campo 6 desde 1989 (figura 3), se observa que, desde el año 1996, sus resultados son similares a la media de Todos los Campos. Sin embargo, como 
FIGURA 2

Porcentaje de éxito en todos los campos (período 1989-2007)

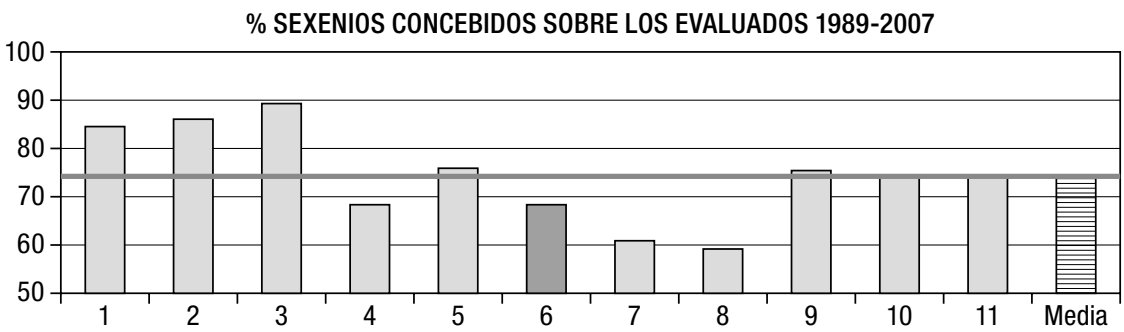

consecuencia de la heterogeneidad detectada tras sucesivas evaluaciones en el campo científico 6, en noviembre de 2006, una Resolución de la CNEAI subdivide este campo en tres subcampos, 6.1 (Tecnologías Mecánicas y de la Producción), 6.2 (Ingenierías de la Comunicación, Computación y Electrónica) y 6.3 (Arquitectura, Ingeniería Civil, Construcción y Urbanismo), cada uno con su correspondiente comité evaluador.

De modo que, a pesar de la bondad aparente de los resultados anteriores considerando el conjunto del campo 6, cuando se particulariza, a partir de 2006, para cada subcampo, se observa que los resultados para el subcampo 6.3 se sitúan un $10 \%$ por debajo de la media nacional y un $20 \%$ respecto del resto de áreas del campo 6.

FIGURA 3

Evolución del éxito en el campo 6 (período 1989-2007)

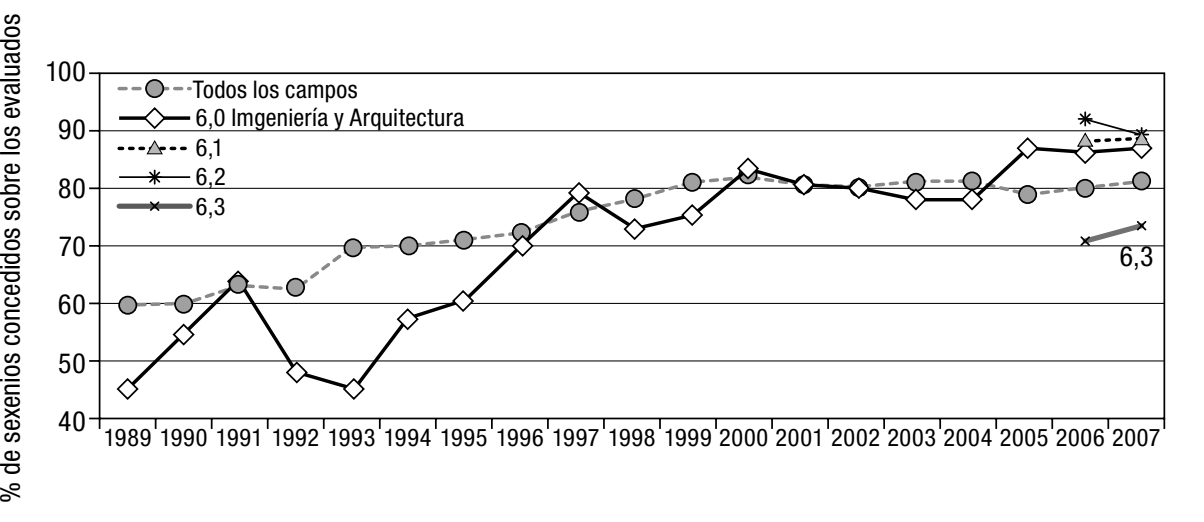


En el apartado siguiente se han ido analizando, progresivamente, con mayor detalle, los datos correspondientes a las 7 áreas del campo 6.3 objeto de estudio, representando mediante gráficas, los valores correspondientes a cada uno de los grupos, tanto en valores absolutos (altura de las barras) como en el porcentaje que representan respecto al colectivo analizado.

\subsection{Evaluación de los resultados de la CNEAI en las áreas relacionadas con la arquitectura}

Los resultados de investigación correspondientes al conjunto de profesores se muestran en la figura 4.a), que representa el número de profesores en valor absoluto, con y sin sexenios y su porcentaje respecto del total de profesores en el conjunto de Todos los Campos. Se observa que un 36\% del profesorado no posee ningún tramo de investigación. Al comparar dicho porcentaje con el correspondiente a las 7 áreas seleccionadas [figura 4.b)] se constata que esta cifra es todavía mayor (69\% de profesorado sin ningún tramo de investigación, bien porque no lo ha solicitado, bien porque se lo han denegado).

Estos porcentajes se ven incrementados para algunas áreas [figura 4.c)], siendo Ingeniería del Terreno el área que menor porcentaje de sexenios tiene (16\%)

\section{FIGURA 4}

Número de profesores con y sin sexenios en valor absoluto y porcentaje que representan sobre el total de profesores en todos los campos $y$ en las 7 áreas estudiadas

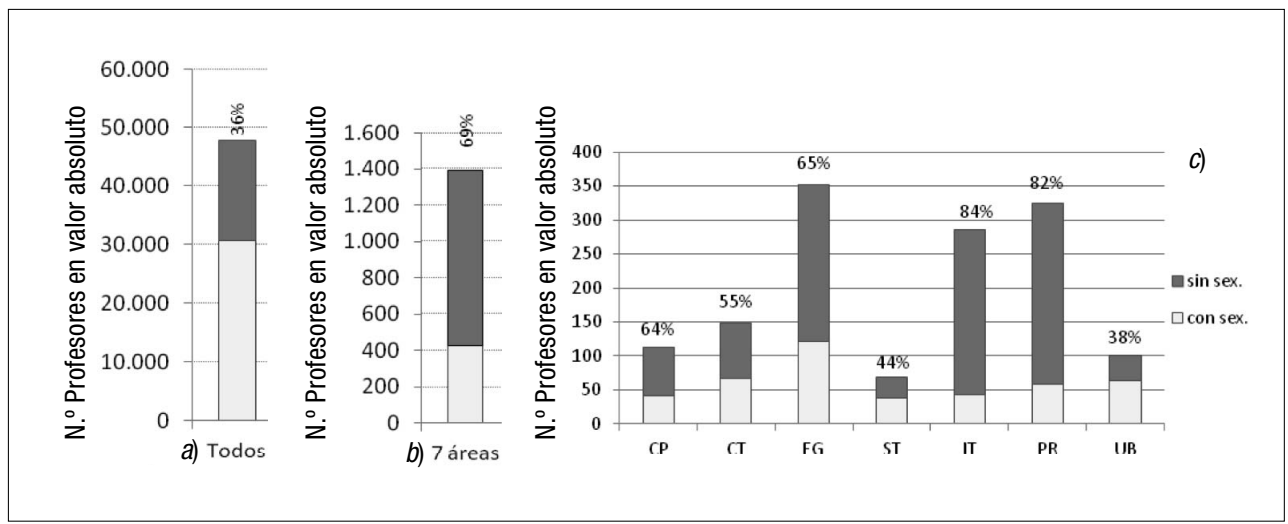

Por ser la evaluación un proceso reglado que se solicita voluntariamente, tal y como se comentó en la introducción, es necesario identificar inicialmente cuál es el conjunto de profesores que han solicitado ser evaluados, con objeto de establecer si, el hecho de que haya tantos profesores sin sexenios en el colectivo 
analizado, se debe a porcentajes de solicitudes bajos o a porcentajes de rechazos altos.

Para ello, se representa en la figura 5 el número de profesores de cada una de las áreas estudiadas, divididos entre los que NO han solicitado la evaluación de su actividad investigadora, NS, y los que Sí lo han hecho, S, (primera columna de cada área) y, de estos últimos, aquellos a los que se les ha concedido al menos un tramo de investigación, C, y a los que se les ha denegado, NC, (segunda columna), además de indicar el porcentaje que representa cada uno de los grupos.

El área de Estructuras, por tener el mayor número de profesores (352), es la que tiene el mayor número de profesores que han solicitado sexenios y sexenios concedidos (233), aunque hay que señalar que, en este área, hay muchos profesores que ni son arquitectos ni están impartiendo docencia en Escuelas de Arquitectura. El porcentaje de profesores que no han solicitado la evaluación (NS) supera, en las 7 áreas analizadas, al de Todos los Campos (23\%), siendo significativamente superior en Construcción y Expresión Gráfica, con un 61\% y $71 \%$ respectivamente.

Por otro lado, el porcentaje de sexenios denegados o no concedidos (NC) con respecto a los solicitados (S) supera en todos los casos (del 18\% al 52\%) al del conjunto de Todos los Campos (17\%), siendo Construcción el área con mayor porcentaje de fracasos con un $52 \%$ de sexenios rechazados mientras Composición tiene el menor valor (18\%).

Por último, se ha reflejado, en cada área, el número de sexenios por profesor con sexenios. Los profesores que más número de sexenios tienen pertenecen a Ingeniería del Terreno, con una media de 2,47 sexenios por profesor, y a Composición, con una media de 2,43, siendo estos últimos los únicos que superan la media de Todos los Campos (2,32). La menor media corresponde a los profesores de Expresión Gráfica, con 1,43 sexenios por profesor.

Cuando se analizan los resultados anteriores, desglosándolos, además de por Áreas, por Cuerpos, figura 6, se deduce que, salvo en Ingeniería del Terreno, los TEU apenas han solicitado sexenios, correspondiendo la mayoría de las solicitudes de sexenios rechazadas a los TU (previsible al ser el colectivo más numeroso, aunque en los datos correspondientes a Todos los Campos este porcentaje es bastante menor).

A continuación, en la figura 7 , se analiza el reparto de los sexenios concedidos en cada uno de los cuerpos por áreas, siendo los CU, TU y TEU del área de Estructuras los que mayor porcentaje de sexenios acumulan, (30\%, 24\% y 31\%) mientras que son los CEU de Construcción e Ingeniería del Terreno los que obtienen los mayores porcentajes (gráficas de la izquierda).

En cuanto al ratio sexenios/profesor en cada uno de los cuerpos, se calculan dos valores (gráfica de la derecha): El ratio 1, que corresponde al número de sexenios concedidos en cada uno de los cuerpos repartidos entre los profesores de dicho cuerpo que han solicitado la evaluación, y el ratio 2, como cociente entre el número de sexenios concedidos a un cuerpo dividido por la totalidad de profesores de dicho cuerpo. 
FIGURA 5

Situación de los profesores en relación a los sexenios (NS, S, NC y C) por áreas

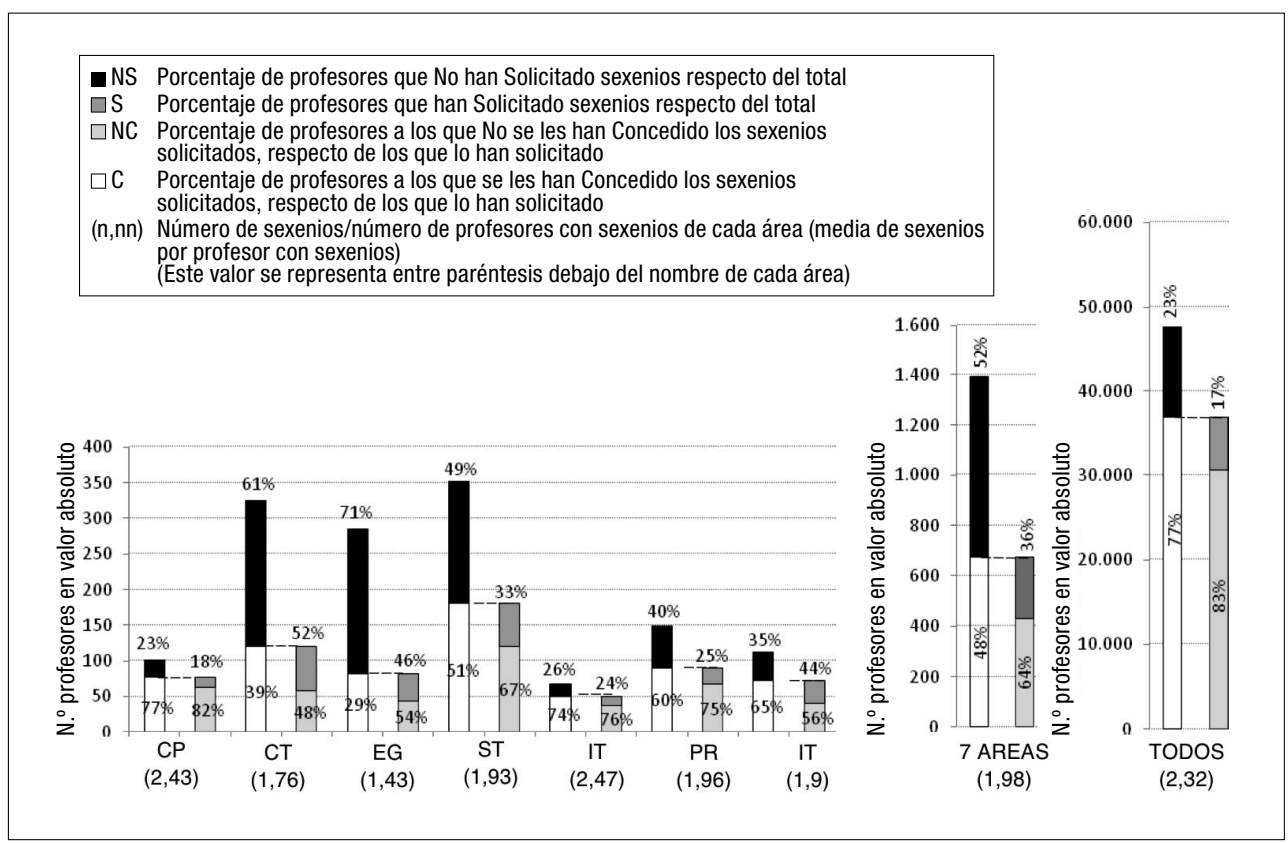

Analizando los resultados obtenidos, se observa que en el caso de los CU el ratio 1 y el ratio 2 tienen, en la mayoría de las áreas, valores bastante similares, lo que significa que la mayor parte de los profesores pertenecientes a este cuerpo ha solicitado la evaluación de la actividad investigadora y ha obtenido un resultado favorable. Se trata, como cabría esperar, del cuerpo con mayor porcentaje de éxito. No obstante, hay que señalar que únicamente en el caso de Composición (ratio 1 igual a 3,2 y ratio 2 igual a 3,1) y de Ingeniería del Terreno (ratio 1 igual a 3,2 y ratio 2 igual a 2,7) las cifras se aproximan a las del conjunto de Todos los Campos (ratio 1 igual a 3,29 y ratio 2 igual a 3,21). En el caso de los TU ya se aprecian diferencias entre los dos ratios, sin embargo, el ratio 1 supera en Composición, Ingeniería del Terreno y Proyectos al de Todos los Campos y se aproxima a su valor en Construcción, Estructuras y Urbanismo. Los ratios correspondientes a los CEU de Ingeniería del Terreno (3,5 y 1,4) y de Urbanismo (2 y 2) superan a los globales (1,04 y 0,76) observándose, como era previsible, que los menores ratios corresponden al cuerpo de TEU.

Estos ratios dan una visión general de los resultados de los profesores de cada uno de los cuerpos en las 7 áreas analizadas. Sin embargo, dado que cada profesor puede obtener hasta 6 sexenios, es necesario estudiar la distribución de los mismos por profesor. 
FIGURA 6

Profesores con sexenios no solicitados, solicitados, rechazados y concedidos por áreas y cuerpos
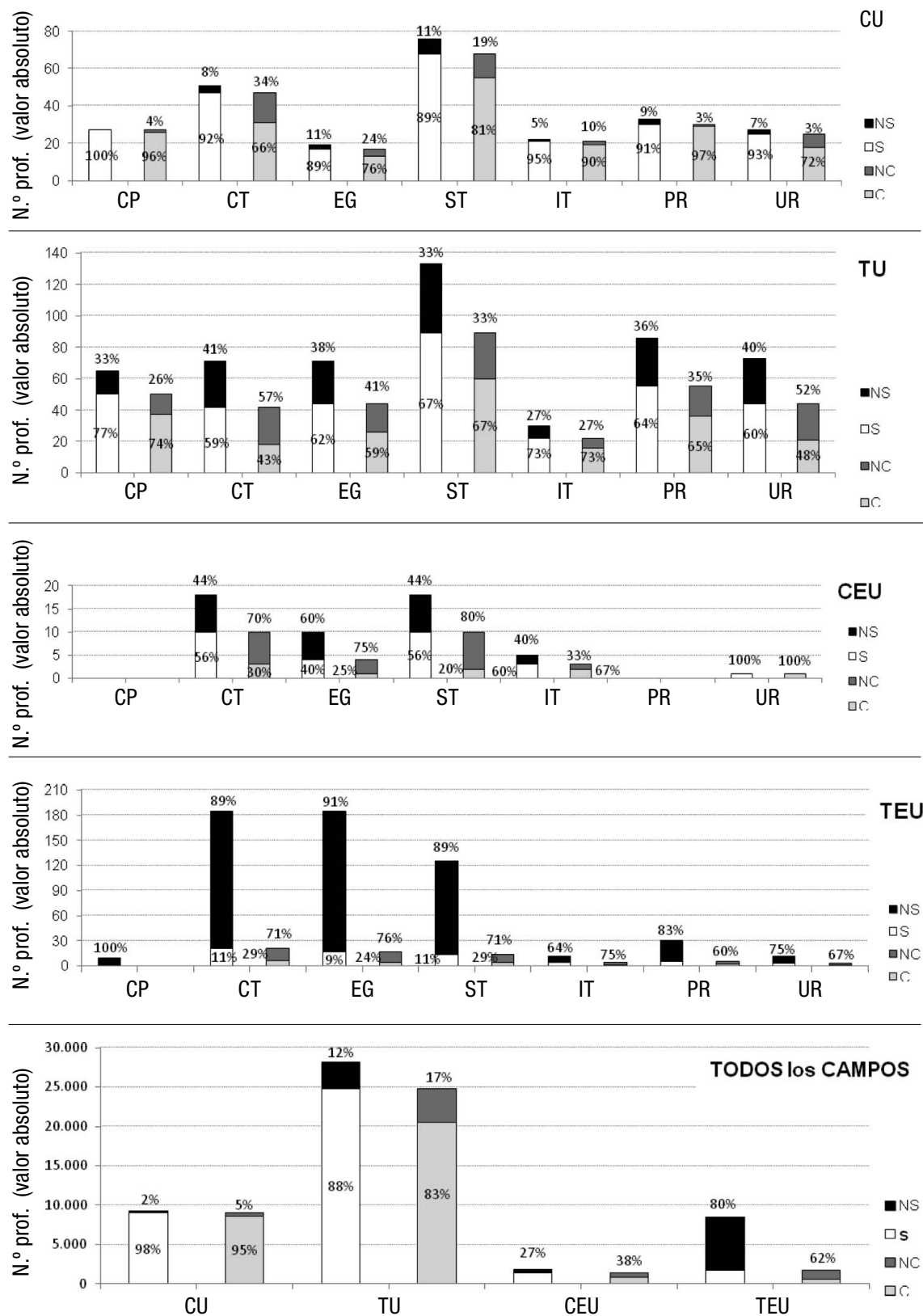
FIGURA 7

Profesores, sexenios y ratios por cuerpos y áreas

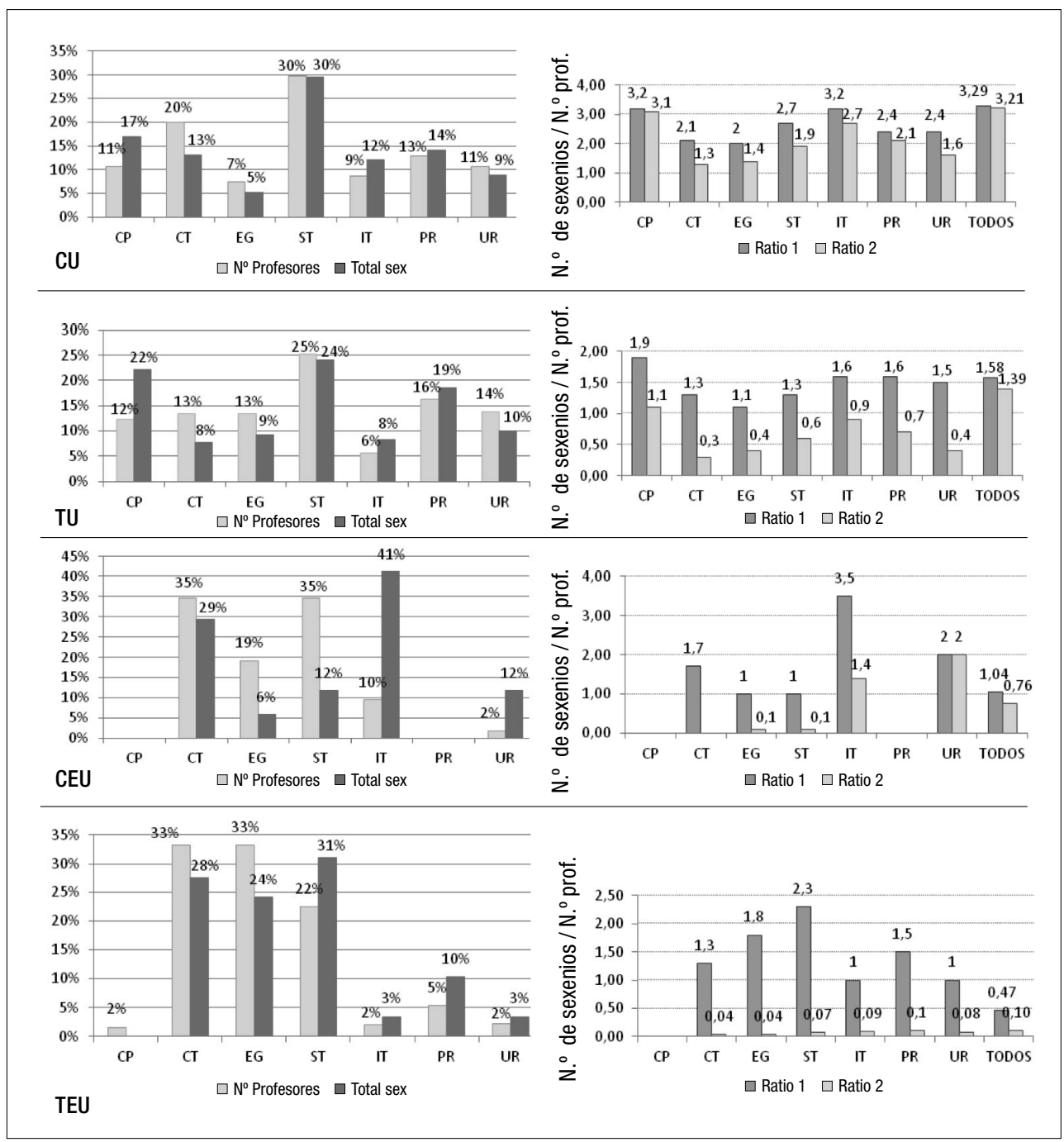

Este análisis tiene por objeto obtener el porcentaje del profesorado perteneciente a cada una de las áreas que ha iniciado con éxito su carrera investigadora (1 sexenio), tiene una trayectoria investigadora consolidada (3 o más sexenios) o ha consolidado los seis posibles tramos de investigación.

Los resultados obtenidos (porcentaje respecto al conjunto de profesores del área y porcentaje respecto de los profesores que han solicitado la evaluación) se muestran en la figura 8. 
FIGURA 8

Distribución de los sexenios por profesores en cada área

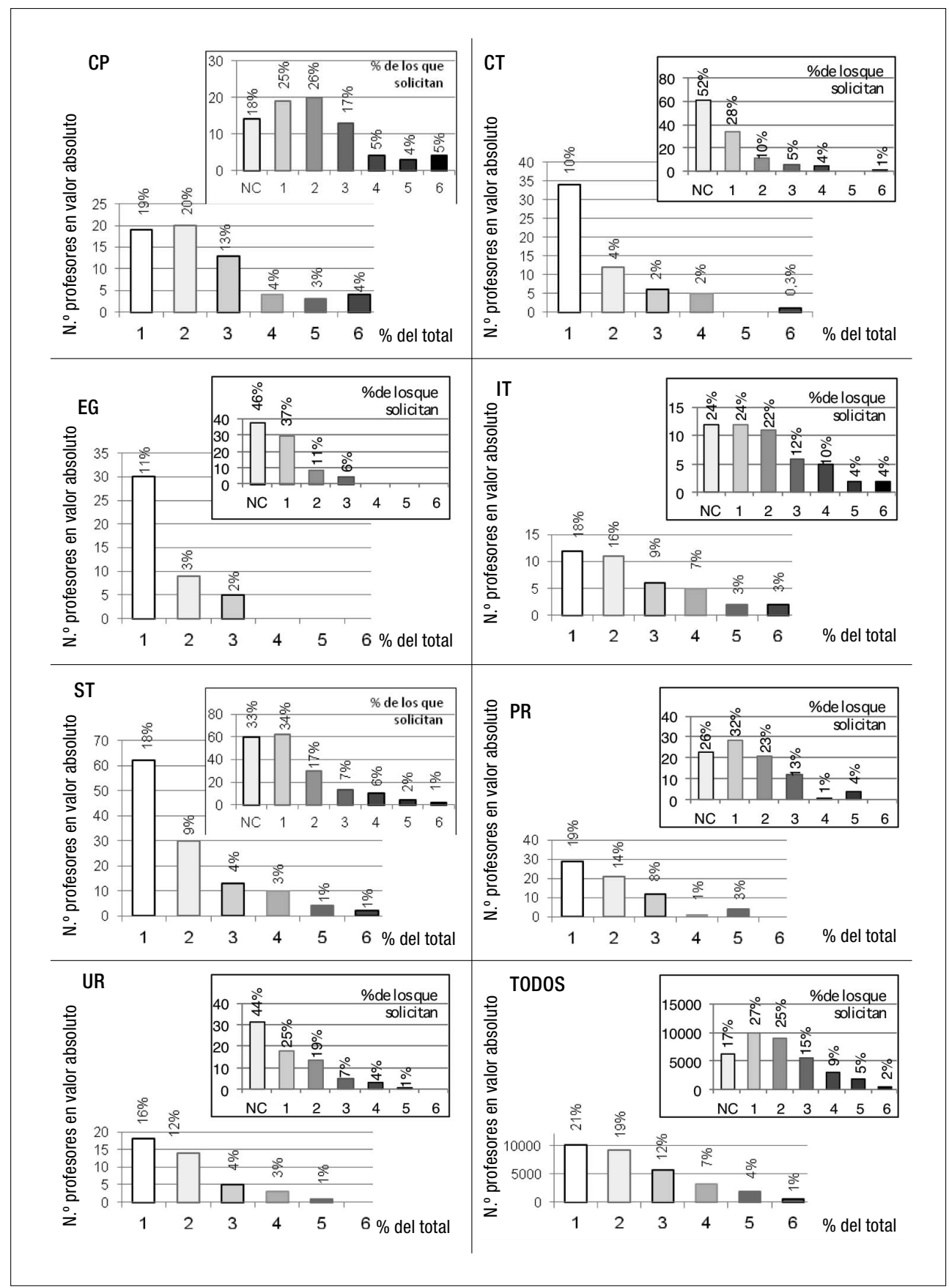


FIGURA 9

Distribución de sexenios por cuerpos en cada área

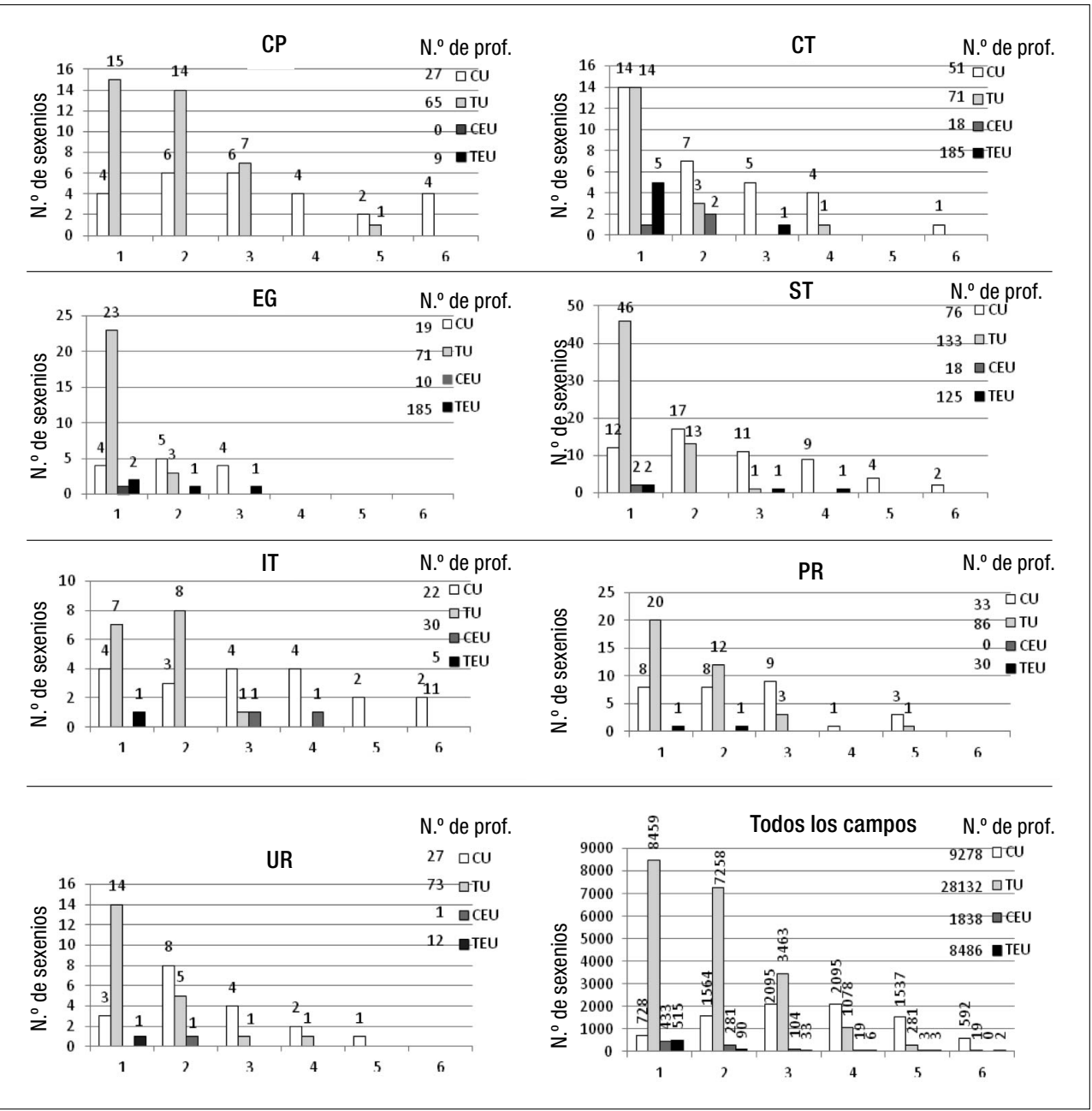

Se constata que Construcción es el área que mayor porcentaje de rechazos tiene $(52 \%)$ y menor proporción de profesores que, con 1 sexenio, apenas han iniciado la carrera investigadora (10\% del total del conjunto de profesores del área o $28 \%$ de los que lo solicitan). También se trata del área con menor porcentaje de profesores con 2, 3, 4, 5 y 6 sexenios.

Por otro lado, Expresión Gráfica y Estructuras son las áreas que mayor porcentaje de profesores con 1 sexenio tienen (37\% y 34\% respectivamente) superior al de Todos los Campos. Estos valores descienden drásticamente cuando se analiza el porcentaje de profesorado de dichas áreas con 2 o más sexenios, lo que 
indicaría que en estas áreas no es fácil consolidar un segundo tramo de investigación.

Finalmente, las áreas de Composición e Ingeniería del Terreno tienen un perfil bastante similar al de Todos los Campos, con un alto porcentaje de investigadores consolidados (con 3 o más sexenios).

Esta información se desglosa por Cuerpos en la figura 9, donde las barras representan, para cada uno de los cuerpos, el número de profesores de cada área que ha consolidado 1, 2, 3, 4, 5 o 6 tramos de investigación. Para tener una idea del porcentaje que representan, se indica, junto a la leyenda, el número de profesores de cada cuerpo.

Sorprende en este análisis que, a excepción del área de Ingeniería del Terreno, apenas haya CEU con sexenios y que algunos TEU, acumulen 3 y 4 sexenios en algunas áreas (Estructuras). Por otro lado, los profesores de Expresión Gráfica son los que menos sexenios acumulan ( 2 o 3, en el mejor de los casos).

Los datos correspondientes a Todos los Campos reflejan que el número de sexenios reconocidos a los TEU es muy reducido. Es de suponer que el temor al rechazo del tramo solicitado, con la correspondiente renuncia de parte de las aportaciones presentadas para la siguiente evaluación, contribuye a que los posibles interesados no hayan solicitado la evaluación.

Por otro lado, no hay que olvidar que el número de sexenios a los que puede optar un investigador está directamente ligado a su edad y al número de años dedicados a dicha actividad. Por este motivo, la información que proporciona el análisis puramente cuantitativo realizado en este apartado es, sin duda, incompleta. Siendo la edad un factor importante para determinar la excelencia de los investigadores, la CNEAI la tiene en cuenta en sus informes por medio de los índices de calidad iE e is, definidos en el apartado 3 y que se analizan a continuación.

\section{3. Índices de calidad}

La CNEAI valora la calidad de la investigación mediante el índice: is (porcentaje de sexenios que los investigadores de un grupo tienen respecto al que deberían poseer, fijando en 27 años la edad promedio de inicio de la publicación de los resultados de la investigación) y el índice iE (porcentaje de investigadores de un grupo que poseen la totalidad de sexenios posibles), siendo los valores próximos a la unidad indicativos de una investigación de excelencia.

En la figura 10 se representan los valores de ambos índices por cuerpos y áreas. El cuerpo con mayor índice is es el de los CU y dentro de éstos los de Composición e Ingeniería del Terreno, como cabía esperar a raíz de los resultados de los apartados anteriores

El índice iE más elevado pertenece a los CU de Ingeniería del Terreno, de los cuales el 31,82\% posee todos los sexenios que les corresponderían por edad, mientras que ninguno de Expresión Gráfica, Estructuras o Composición los tiene. En conjunto Ingeniería del Terreno es el área que mejores índices de calidad posee. 
FIGURA 10

Índices de calidad de la investigación por cuerpos y áreas

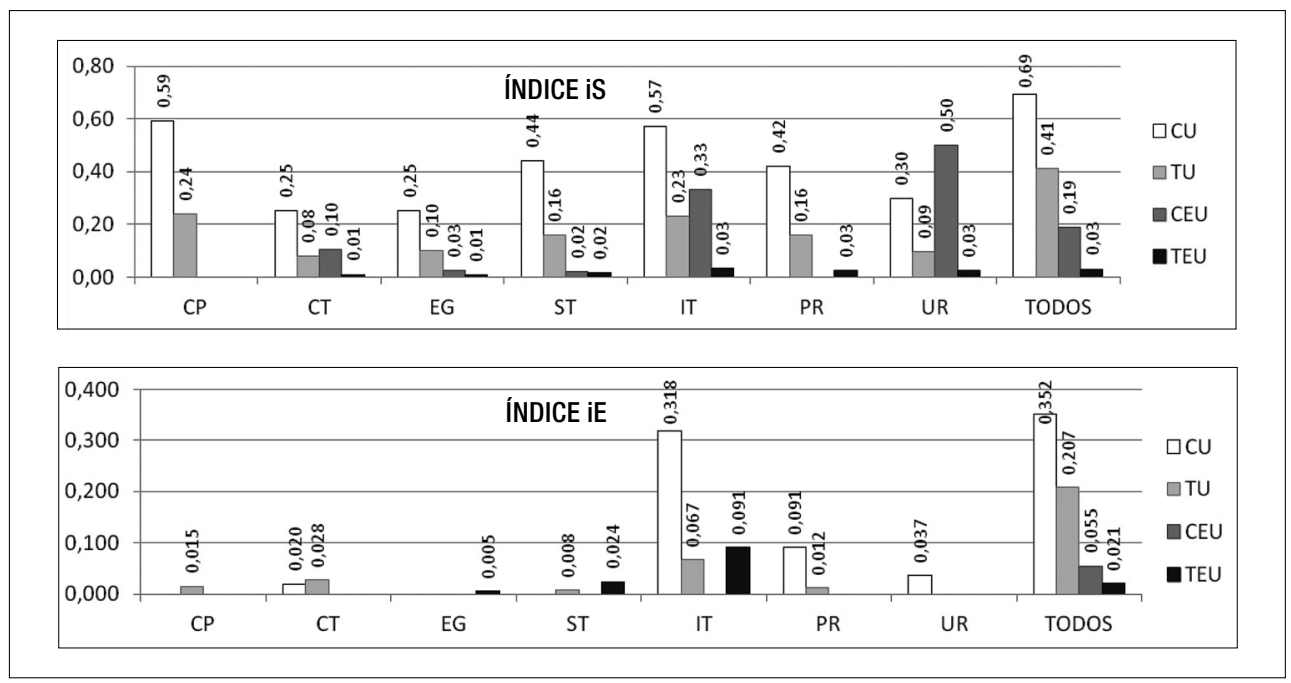

Considerando que el investigador de excelencia es aquel que ha consolidado los tramos de investigación que le corresponden por edad, en la figura 11 se representa dicho colectivo dentro del conjunto del profesorado de las 7 áreas analizadas.

FIGURA 11

Investigadores de excelencia por cuerpos y áreas

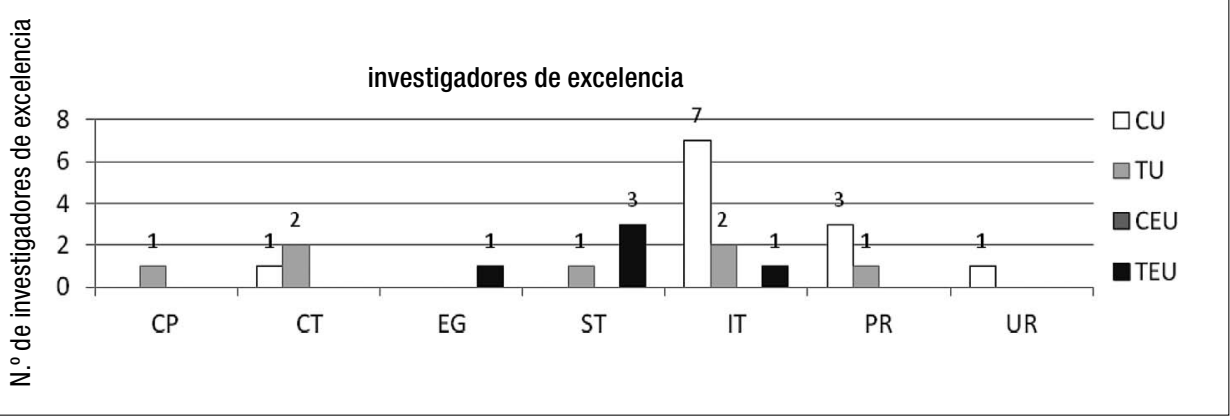

En esta gráfica se observa que:

- Sólo 24 profesores de los 1393 considerados se pueden calificar como investigadores de excelencia, es decir, el 1,7\% del conjunto del profesorado analizado. 
- Hay al menos uno de estos investigadores de excelencia en cada una de las 7 áreas consideradas, no siendo necesariamente CU, como sería de esperar.

- Sorprendentemente 5 de ellos son TEU (20\% de los investigadores de excelencia del conjunto de las 7 áreas) y ninguno CEU.

\section{Discusión y conclusiones}

El análisis de los resultados obtenidos en cada una de las áreas objeto de estudio evidencia una gran disparidad entre ellas, siendo las áreas de Composición e Ingeniería del Terreno las que mejores resultados tienen, situándose en el polo opuesto las áreas de Construcción y Expresión Gráfica, con los peores resultados. Los buenos resultados del área de Composición vendrían a confirmar la tesis de Javier Monedero que considera que «la historia» es el área más representativa de la investigación en el ámbito de la arquitectura. En cuanto a la Ingeniería del Terreno, se trata de un área a la que se dedican básicamente los ingenieros, representando los arquitectos una minoría.

Por otra parte, el porcentaje de rechazos correspondiente a las 7 áreas en conjunto es del 36\%, (oscilando desde un 52\% y 46\%, respectivamente, para las áreas de Construcción y Expresión Gráfica hasta un 18\% y 24\%, respectivamente, para las áreas de Composición e Ingeniería del Terreno) superándose en las siete áreas la media de todos los campos (17\%). Estos porcentajes de rechazos tan altos son bastante preocupantes ya que parece razonable que los solicitantes, conocedores de los criterios de evaluación, soliciten ser evaluados cuando consideren que el resultado de la evaluación va a ser positiva, entre otras cosas porque, caso de ser negativa, deben, además de renunciar a parte de las aportaciones presentadas, esperar tres años para volver a solicitarla. En general, estos porcentajes son menores para los catedráticos de universidad, y van aumentando a medida que se desciende en el escalafón.

En cuanto a la cantidad de sexenios acumulados por CU, TU, CEU y TEU, los índices de calidad de la CNEAI y el número de investigadores de excelencia de cada una de las 7 áreas consideradas de arquitectura y urbanismo se alejan bastante, con pequeños matices entre áreas, de la media de Todos los Campos, tal y como se ha mostrado en las gráficas de las figuras 5 a 11.

Los resultados anteriores justificarían el descontento general de los profesores de la rama de arquitectura y urbanismo, habiéndose convertido la evaluación de la investigación en un tema prioritario para este colectivo.

Por otro lado no se puede ignorar el alto porcentaje de profesores del conjunto de las 7 áreas analizadas que no han solicitado la evaluación (52\% frente al $23 \%$ de todos los campos), porcentajes que a su vez son bastante dispares entre áreas (61\% y 73\% en Construcción y Expresión Gráfica frente a 23\% y 26\% en Composición e Ingeniería del Terreno) y cuerpos: (11\% como máximo en CU y $64 \%$ como mínimo en TEU). 
Las razones por las que los profesores universitarios de la rama de arquitectura y urbanismo no solicitan la evaluación podrían ser bien la inexistencia de actividad investigadora bien la consideración de que la actividad investigadora que desarrollan no coincide con la reconocida por la CNEAI.

En cuanto al primer supuesto, es bastante posible que, en el momento en que se aprobó la LOU, los profesores de las escuelas de arquitectura no desarrollaran ninguna actividad científica, sobre todo teniendo en cuenta que han compaginado, tradicionalmente, la docencia y el desarrollo de la actividad profesional, siendo esta última la que les mantenía en contacto con las últimas técnicas constructivas, los nuevos materiales, y la realidad construida, permitiéndoles experimentar e innovar y, finalmente, transmitir el conocimiento adquirido y renovado por medio de su actividad docente. Sin embargo, se entiende que, actualmente, la mayoría de los profesores funcionarios de las escuelas de arquitectura, por su condición de PDI (Personal docente e investigador) deberían realizan alguna actividad científica, al menos tras la aprobación de la LOU y su nueva política de calidad.

Partiendo, por tanto, de la hipótesis (no contrastada) de que los profesores arquitectos realizan alguna actividad científica, se podría afirmar, a la vista de los resultados anteriores, que es fundada la sensación generalizada entre este colectivo de la falta de sintonía entre los criterios de la CNEAI y la actividad investigadora de los arquitectos. Esta situación, justificaría el porcentaje tan bajo de evaluaciones solicitadas, por un lado, y el alto porcentaje de rechazos por otro, todo ello a pesar de que el área de la Arquitectura, incluida inicialmente en el campo 6 junto con las Ingenierías, posee desde el año 2006, con objeto de considerar su peculiaridad como campo científico, su propio comité (subcampo 6.3) y criterios de evaluación propios, sin cambios desde entonces hasta la fecha.

En los criterios específicos del subcampo 6-3, se puede observar que, además de los trabajos publicados en revistas de reconocida valía, es posible considerar como aportaciones valorables los desarrollos tecnológicos o arquitectónicos relevantes así como los proyectos innovadores de Arquitectura y Urbanismo, las patentes, los libros y las actas de congresos. Sin embargo, a la luz de los resultados analizados, parece que la inclusión de los desarrollos tecnológicos o arquitectónicos y los proyectos innovadores de Arquitectura y Urbanismo como aportación a considerar no ha conseguido mejorar sustancialmente los resultados (sólo 24 investigadores de excelencia en 2008 en las 7 áreas consideradas), seguramente debido a que estos proyectos no están al alcance de todos los arquitectos, no siendo fácil que ningún promotor, público o privado, esté dispuesto a financiar la experimentación que aporte nuevo conocimiento. Por otro lado, cuando se considera que un proyecto arquitectónico es una aportación evaluable por su singularidad y carácter innovador, debería valorarse positivamente cada una de las facetas desde su concepción hasta su materialización: sistemas y técnicas de representación, composición, proceso de diseño, sistemas estructurales y métodos de cálculo, instalaciones, materiales, técnicas constructivas, sostenibilidad, eficien- 
cia, impacto y repercusión social, etc., por ser partes de un todo indivisible que es el proyecto arquitectónico. Sin embargo, habitualmente, se valora únicamente la aportación del autor del mismo, que suele ser de tipo compositivo o proyectual, dejando de lado la aportación del resto de profesionales implicados.

En cuanto a los trabajos publicados en revistas de reconocida valía, el incluir las revistas recogidas en bases de datos internacionales de ingeniería (como, por ejemplo, TRIS Electronic Bibliography data e International Development Abstracts), los índices internacionales de publicaciones de arquitectura (Avery Index to Architectural Periodicals de la Avery Library —Columbia University-; Architectural Publications Index del Royal Institute of British Architecs; Arts and Humanities Citation Index - ISI-). Además de las que ocupan posiciones relevantes en el "Subject Category Listing" del "Journal Citation Reports» del «Science Citation Index»o del "Journal Citation Reports» del "Social Sciences Citation Index» no parece haber sido una medida suficiente. En esa línea viene manifestándose el profesorado de las escuelas de arquitectura en las sucesivas Jornadas sobre Investigación en Arquitectura y Urbanismo celebradas en 2004, 2006, 2009 y 2011, con el pleno apoyo de la Conferencia de Directores de Escuelas de Arquitectura de España, que, tal y como se mencionó en la introducción, se encuentra trabajando entre otras cosas, en la elaboración de unos indicadores de calidad de la actividad científica de sus profesores, con objeto de ajustar los criterios del subcampo 6.3 y acercar los resultados de las evaluaciones a la realidad de la investigación realizada por este colectivo.

Con ese mismo objetivo, el Servicio de Bibliotecas y Documentación de la UPC tras el encargo de la Oficina Técnica de Investigación, Desarrollo e Innovación de su universidad ha elaborado un listado de revistas especializadas en Arquitectura y Urbanismo llevando a cabo su posterior evaluación, para poder utilizarlo como base para la revisión del sistema de indicadores de la actividad investigadora en dicho ámbito (Viñas-Cullell y Minobis-López, 2007).

Por último, no se puede concluir este trabajo sin subrayar que, desde 2007, poseer un sexenio se ha convertido en un requisito imprescindible para poder participar en las actividades docentes, investigadoras y de gestión en la Universidad, y que el 69\% del colectivo analizado no tenía, hasta la fecha del informe de la CNEAI, ningún tramo de investigación consolidado, siendo bastante difícil progresar académicamente en esta situación, destacando el hecho de que el $40 \%$ del profesorado de las 7 áreas objeto de estudio son TEU, cuerpo a extinguir que necesita ver reconocida su actividad investigadora para poder promocionar a TU.

Finalmente, para ver si los últimos esfuerzos han mejorado la situación o si, por el contrario, tal y como sigue pensando el colectivo, es urgente un replanteamiento del sistema de evaluación que ajuste todavía más y mejor los mecanismos de acreditación, estableciendo nuevos índices de calidad aplicables a las publicaciones e incluyendo, al mismo tiempo, todos los matices específicos del campo de la Arquitectura y el Urbanismo, es imprescindible disponer de los resultados de la última convocatoria. 


\section{Bibliografía}

Buela-Casal, G. (2003): Evaluación de la calidad de los artículos y de las revistas científicas: Propuesta del factor de impacto ponderado y de un índice de calidad. Psicothema, vol. 15 (1), 23-35.

CNEAI (2005): Memoria de los resultados de las evaluaciones realizadas de 1989 a 2005 (profesores de universidad). Ministerio de Educación y Ciencia. Disponible en: http:// www.educacion.es/dctm/ministerio/horizontales/ministerio/organismos/cneai/2005memoria-1989-2005-universidad.pdf [fecha de consulta: 19/01/2011].

CNEAI (2009): Informe sobre los resultados de las evaluaciones de la CNEAI. La situación en 2009. Ministerio de Educación y Ciencia. Disponible en: http://www.educacion.es/ $\mathrm{dctm} / \mathrm{ministerio/horizontales/ministerio/organismos/cneai/2009-info-v5.pdf} \mathrm{[fecha} \mathrm{de}$ consulta 19/01/2011].

CNEAI (2009): Informe 2009. Tabla de resultados por Áreas. Ministerio de Educación y Ciencia. Disponible en: http://www.educacion.es/dctm/ministerio/horizontales/ministerio/organismos/cneai/2009-tabla-areas.xls [fecha de consulta: 19/01/2011].

CNEAI (2009): Resultados de la evaluación por campos científicos. Evaluación 2006 y Evaluación 2007. Ministerio de Educación y Ciencia. Disponible en: http://www.educacion.es/dctm/ministerio/horizontales/ministerio/organismos/cneai/2009-resultadosevaluacion-06.pdf http://www.educacion.es/dctm/ministerio/horizontales/ministerio/ organismos/cneai/2009-resultados-evaluacion-07.pdf [fecha de consulta: 19/01/2011].

De las Heras, G., y Polo, G. (2009): La evaluación de la investigación universitaria en ciencias jurídicas en el marco general español. Revista de la Educación Superior, vol. XXXVIII (2), 63-79.

Delgado-López-Cózar, E. (2009): Claroscuros en la evaluación científica en España. Jornada Medes 2009. Disponible en: http://ec3.ugr.es/publicaciones/BoletinMEDES10.pdf.

Fayos-Vallés, F. (2005): Valoración de la actividad investigadora de los profesores del ámbito de la Arquitectura y el Urbanismo (AU). Disponible en: http://congreso.us. es/iau04/DATOS/ValoracionActividadInvestigacionArqyUrb.pdf [fecha de consulta: 10/01/2011].

Font-Comas, J. (2006): Indicadores de la calidad. Segundas jornadas sobre investigación en Arquitectura y Urbanismo, San Cugat del Vallés, 21-23 septiembre 2006. Disponible en: http://upcommons.upc.edu/revistes/bitstream/2099/2211/1/IAU-00027-10.pdf [fecha de consulta: 13/09/2011].

IAU (2006): Conclusiones de las Segundas jornadas sobre investigación en Arquitectura y Urbanismo. San Cugat del Vallés, 21-23 septiembre 2006. Disponible en: http://upcommons.upc.edu/revistes/bitstream/2099/2331/1/Conclusiones.pdf [fecha de consulta: $18 / 05 / 2011]$.

Jiménez-Contreras, E.; Moya-Anegón, F. D., y Delgado-López-Cózar, E. (2003): The evolution of research activity in Spain: The impact of the National Commission for the Evaluation of Research Activity (CNEAI). Research Policy, vol. 32 (1), 123-142.

Jiménez-Contreras, E.; Robinson-García N., y Cabezas-Clavijo, A. (2011): Productividad e impacto de los investigadores españoles: umbrales de referencia por áreas científicas. Revista española de Documentación Científica, vol. 34 (4), 505-526. 
Monedero-Gil, J. (2004): La investigación en arquitectura. Datos para un debate. Primeras Jornadas sobre investigación en arquitectura. Información, crítica y evaluación, Sevilla, 23-25 de septiembre, 2004.

Pinón-Pallarés, H. (2007): Ingeniería civil y arquitectura: investigar para construir. Universia. Disponible en: http://noticias.universia.es/ciencia-nn-tt/reportaje/2007/ 11/12/649848/ingeniería-civil-arquitectura-investigar-construir.html. http://contenidos. universia.es/especiales/entrevistas/civil-arquitectura/helio-pinyon.pdf [fecha de consulta $27 / 05 / 2011]$.

Ruiz-Pérez, R.; Delgado-López-Cózar, E, y Jiménez-Contreras, E. (2010): Principios y criterios utilizados en España por la Comisión Nacional Evaluadora de la Actividad Investigadora (CNEAI) para la valoración de las publicaciones científicas: 1989-2009. Psicothema, vol. 22 (4), 898-908.

Sanz-Menéndez, L. (1995): Research actors and the state: research evaluation and evaluation of science and technology policies in Spain. Research Evaluation, vol. 5 (1), 79-88.

Viñas-Cullell, A., y Minobis-López, E. (2007): La evaluación de las revistas en la investigación en arquitectura y urbanismo. Disponible en: http://bibliotecnica.upc.es/abba/ jornadas/leon/Comunicaciones/Eduad_Minobis_Evaluacion_Revistas_ABBA_2007.pdf [fecha de consulta 27/05/2011].

4IAU+i (2011): IV jornadas Internacionales sobre investigación en Arquitectura y Urbanismo, Valencia, 1-3 junio 2011. Disponible en http://4iau.webs.upv.es/index.html [fecha de consulta 20-05-2011]. 